OPEN ACCESS

Edited by:

Detlev Boison

Legacy Health, United States

Reviewed by:

Hong Qing,

Beijing Institute of Technology, China Subashchandrabose Chinnathambi, National Chemical Laboratory (CSIR),

India

*Correspondence.

Charlie Xiang

cxiang@zju.edu.cn

Received: 07 January 2018 Accepted: 06 April 2018 Published: 24 April 2018

Citation:

Zhao Y, Chen X, Wu Y, Wang Y, Li Y and Xiang $C$ (2018) Transplantation of Human Menstrual Blood-Derived Mesenchymal Stem Cells Alleviates Alzheimer's Disease-Like Pathology in APP/PS1 Transgenic Mice. Front. Mol. Neurosci. 11:140. doi: 10.3389/fnmol.2018.00140

\section{Transplantation of Human Menstrual Blood-Derived Mesenchymal Stem Cells Alleviates Alzheimer's Disease-Like Pathology in APP/PS1 Transgenic Mice}

\author{
Yongjia Zhao, Xin Chen, Yichen Wu, Yanling Wang, Yifei Li and Charlie Xiang*
}

State Key Laboratory for Diagnosis and Treatment of Infectious Diseases, Collaborative Innovation Center for Diagnosis and Treatment of Infectious Diseases, The First Affiliated Hospital, College of Medicine, Zhejiang University, Hangzhou, China

Extracellular $\beta$-amyloid (A $\beta$ ) plaques and neurofibrillary tangles (NFTs) are the pathological hallmarks of Alzheimer's disease (AD). Mesenchymal stem cells (MSCs) have shown therapeutic efficacy in many neurodegenerative diseases, including AD. Human menstrual blood-derived stem cells (MenSCs) are a novel source of MSCs advantageous for their higher proliferation rate and because they are easy to obtain without ethical concerns. Although MenSCs have exhibited therapeutic efficacy in some diseases, their effects on AD remain elusive. In the present study, we showed that intracerebral transplantation of MenSCs dramatically improved the spatial learning and memory of APP/PS1 mice. In addition, MenSCs significantly ameliorated amyloid plaques and reduced tau hyperphosphorylation in APP/PS1 mice. Remarkably, we also found that intracerebral transplantation of MenSCs markedly increased several $A \beta$ degrading enzymes and modulated a panel of proinflammatory cytokines associated with an altered microglial phenotype, suggesting an A $\beta$ degrading and anti-inflammatory impact of MenSCs in the brains of APP/PS1 mice. In conclusion, these findings suggest that MenSCs are a promising therapeutic candidate for AD.

Keywords: Alzheimer's disease, human menstrual blood-derived mesenchymal stem, amyloid- $\beta$, microglia, tau

\section{INTRODUCTION}

Alzheimer's disease (AD) is the most prevalent type of dementia. AD is clinically characterized by progressive memory loss and cognitive dysfunction and neuropathologically characterized by extracellular deposition of amyloid beta $(\mathrm{A} \beta)$ plaques and intracellular neurofibrillary tangles (NTFs) composed of hyperphosphorylated tau proteins (Alzheimer's Association, 2011; Ballard et al., 2011). A $\beta$ is a proteolytic product of the transmembrane amyloid precursor protein (APP), and tau is a microtubule-associated protein that is enriched in neuronal axons and plays a pivotal role in axonal transport. In $A D$, extracellular accumulation of $A \beta$ causes the formation of $A \beta$ plaques, and tau becomes hyperphosphorylated, with consequent misfolding to further form NTFs. Several lines of evidence indicate that the deposition of $A \beta$ aggravates phosphorylated 
tau and affects the surrounding central nervous system (CNS) resident cells, such as microglia, oligodendrocytes and neurons, ultimately causing neuroinflammation, neurodegeneration, and neuronal loss in AD pathologies (Götz et al., 2001; Cunningham, 2013; Zempel et al., 2013).

However, there are no effective therapies for $\mathrm{AD}$ involving pharmacological agents that target either $A \beta$ or tau aggregation inhibitors (Doody et al., 2014; Salloway et al., 2014; Lovestone et al., 2015; Gauthier et al., 2016). Therefore, it is urgent to find alternative therapeutic strategies to treat $\mathrm{AD}$. In recent years, with the development of stem cell technologies, mesenchymal stem cells (MSCs) with self-renewal, multipotency and cytokine secretion properties have been considered as a promising treatment for several neurodegenerative disorders, including AD. Several studies have revealed a beneficial effect of different types of MSCs on disease pathology and cognitive function in AD models. It has been reported that MSCs from bone marrow (BM-MSCs), umbilical cord (UC-MSCs) and adipose tissue (ASCs) can reduce $\mathrm{A} \beta$ deposition and tau hyperphosphorylation and improve impaired spatial memory, and the underlying mechanism of these beneficial effects has been suggested to be associated with microglia (Lee et al., 2010; Lee H.J. et al., 2012).

Human menstrual blood-derived stem cells (MenSCs) are isolated from the menstrual blood of women. MenSCs are a novel type of MSCs that are highly proliferative and multipotent; they exhibit beneficial phenotypes and properties and can also differentiate into the three germ lineages (Meng et al., 2007; Allickson and Xiang, 2012; Sugawara et al., 2014). Compared with other types of MSCs, MenSCs show higher proliferation rates and can be made readily to available through a safer and simpler route without inducing pain or being associated with ethical issues. Furthermore, MenSCs exhibit remarkable regenerative capacity and low immunogenicity (Khoury et al., 2014; Lai et al., 2015). These characteristics make MenSCs a potential therapeutic cell type for use in several disease models, such as type 1 diabetes, myocardial infarction, fulminant hepatic failure, acute lung injury and liver fibrosis (Wu et al., 2014; Zhang et al., 2013; Chen et al., 2017a,b; Xiang et al., 2017). Moreover, transplantation of human endometrial-derived stem cells was reported to restore dopamine production in a Parkinson's disease model (Wolff et al., 2011). Thus, transplantation of MenSCs may be a potential therapeutic strategy for $\mathrm{AD}$.

In the present study, we examined whether intracerebral transplantation of MenSCs could have beneficial effects on the neuropathology of AD in APP and presenilin one (PS1) double-transgenic mice. We discovered that MenSCs reduced A $\beta$ deposition and tau hyperphosphorylation, improved cognitive decline, modulated microglia activation and restored $\mathrm{A} \beta$ clearance capacity in APP/PS1 transgenic mice.

\section{MATERIALS AND METHODS}

\section{Animals}

Seven-month-old double-transgenic APPswe/PSEN1dE9 mice were used in this study. APPswe/PSEN1dE9 mice with a C57BL/6 background overexpress the mutant human genes APPswe
(Swedish mutations K594N/M595L) and presenilin-1 with the exon-9 deletion (PS1-dE9). We used only male mice because of the gender-specific differences in the progression of $\mathrm{AD}$ pathology. APPswe/PSEN1dE9 mice and 7-month-old C57BL/6J mice were obtained from Nanjing BioMedical Research Institute of Nanjing University (Nanjing, China). Mice were housed under standard conditions including a 12-h light/dark cycle and allowed free access to food and water at the Laboratory Animal Center of Zhejiang University. All animal experiments were approved by the Animal Care and Use Committees of Zhejiang University.

\section{Cell Isolation and Culture}

Human menstrual blood-derived stem cells were isolated and cultured as described previously (Meng et al., 2007; $\mathrm{Wu}$ et al., 2014). The volunteer donors provided informed consent, and the isolation procedure was approved by the Ethics Committee of the First Affiliated Hospital, College of Medicine, Zhejiang University, China. Briefly, menstrual blood samples were collected with a DivaCup (Kitchener, ON, Canada) from healthy women. Mononuclear cells were separated by density gradient centrifugation with Ficoll-Paque (Thermo Fisher Scientific, MA, United States). The interlayer cells were then collected and cultured in Chang medium (S-Evans Biosciences, Hangzhou, China). Cells were cultured in a tissue culture flask (Corning, Corning, NY, United States) at $37^{\circ} \mathrm{C}$ in a $5 \% \mathrm{CO}_{2}$ humidified atmosphere. The medium was changed every 3 days and subcultured by using 0.25\% trypsin EDTA (Thermo Fisher Scientific) until reaching approximately 70-80\% confluence. The MenSCs used in the experiments were from passages 3 to 5 .

\section{Characterization of the MenSCs}

The surface markers of the MenSCs were evaluated using fluorescence-activated cell sorting. In brief, $1 \times 10^{6}$ cells were collected and resuspended in staining buffer. MenSCs were then incubated with antibodies including CD29, CD34, CD45, CD73, CD90, CD105, CD117, human leukocyte antigen-DR (HLA-DR) and isotype control (BD, Franklin Lakes, NJ, United States) in the dark for $20 \mathrm{~min}$. Stained cells were resuspended in staining buffer and analyzed with an FC 500 flow cytometer (Beckman, Brea, CA, United States).

\section{Transplantation of MenSCs in APP/PS1 Transgenic Mice}

Human menstrual blood-derived stem cell suspensions or phosphate-buffered saline (PBS) alone were transplanted into APP/PS1 transgenic mice at the age of 7 months. Mice were anesthetized with isoflurane and fixed on a stereotaxic apparatus (RWD Life Science, Shenzhen, China). Using a 10- $\mu$ l syringe (Hamilton) and an automated syringe pump (KD scientific, United States), $3 \mu \mathrm{l}$ (approximately $1 \times 10^{5}$ cells) of MenSC suspension was injected at a rate of $0.3 \mu \mathrm{l} / \mathrm{min}$ bilaterally into the hippocampus according to the following stereotaxic coordinates: $1.9 \mathrm{~mm}$ posterior to the bregma, $1.2 \mathrm{~mm}$ bilateral to the midline and $1.7 \mathrm{~mm}$ ventral to the skull surface. The syringe was kept in place for $5 \mathrm{~min}$ after the injection. 


\section{Morris Water Maze}

We used the Morris water maze to examine the spatial learning and memory function of APP/PS1 transgenic mice. Briefly, a white tank was filled with water to a depth of $20 \mathrm{~cm}$. Opaque paint was added to the water before the tests. A circular platform $(10 \mathrm{~cm}$ in diameter) was located at a fixed position approximately $1 \mathrm{~cm}$ below the water surface. One day before escape latency training, all mice were habituated to the maze. In escape latency training, the animals were subjected to four trials each day for six consecutive days. In each of the four trials, mice were placed in the water at four different starting positions equally spaced around the perimeter of the pool, and the mouse was given $60 \mathrm{~s}$ to swim to find the platform. If the mouse did not find the platform within $60 \mathrm{~s}$, the mouse was guided to the platform and was allowed to remain on the platform for $10 \mathrm{~s}$. The time the mice spent to find the platform was measured as the escape latency. On the 7 th day, the platform was removed from the pool in the probe trial. Each animal was subjected to one trail and the number of times the mouse crossed the previous location of the platform in one minute was recorded.

\section{Tissue Preparation}

Mice were anesthetized with $1 \%$ pentobarbital sodium after behavioral experiments and immediately transcardially perfused with cold saline and $4 \%$ paraformaldehyde fixative in $\mathrm{PBS}(\mathrm{pH}$ 7.4). The brain was removed and post-fixed for $24 \mathrm{~h}$ at $4^{\circ} \mathrm{C}$ and then incubated consecutively in 20 and $30 \%$ sucrose solutions at $4^{\circ} \mathrm{C}$. Sequential $30-\mu \mathrm{m}$ coronal sections were prepared by using a cryostat (CryoStar NX50, Thermo Scientific) and stored at $-20^{\circ} \mathrm{C}$.

\section{Thioflavin S Staining}

The brain sections were incubated with $1 \%$ thioflavin S solution dissolved in distilled water containing 50\% ethanol for $5 \mathrm{~min}$ and differentiated in $50 \%$ ethanol three times. Fluorescence imaging was visualized by using an OLYMPUS IX83-FV3000OSR (Olympus Corporation, Japan). To quantify the plaque load, the plaque areas of five sections of the cortex and hippocampus of the mice in each group were calculated and analyzed.

\section{Immunofluorescence}

The brain sections were incubated for $1 \mathrm{~h}$ in PBS containing $10 \%$ normal goat serum and $0.1 \%$ Triton X-100 at room temperature. The brain sections were then incubated with primary antibodies overnight at $4^{\circ} \mathrm{C}$. The following primary antibodies were used: mouse anti-6E10 monoclonal antibody (1:500; Covance, Princeton, NJ, United States) and rabbit antiIba-1 polyclonal antibody (1:500; Wako Chemicals, Japan). The brain sections were then rinsed in PBS and incubated with the following secondary antibodies: Alexa Fluo-488conjugated goat anti-rabbit IgG antibody (1:500; Abcam) and Alexa Fluo-635-conjugated goat anti-mouse IgG antibody (1:500; Invitrogen). Finally, the brain sections were visualized using an OLYMPUS IX83-FV3000-OSR (Olympus Corporation, Japan).

\section{Western Blot Analysis}

Hippocampal and cortical tissues were dissected and homogenized in RIPA buffer (Beyotime Biotechnology) supplemented with a cocktail of protease (Sigma) and phosphatase inhibitors (Thermo Fisher Scientific). The lysate was centrifuged at $12000 \times g$ for $15 \mathrm{~min}$ at $4^{\circ} \mathrm{C}$. Protein concentration was determined using a bicinchoninic acid protein assay kit (Beyotime Biotechnology). Equal amounts of total protein $(50 \mu \mathrm{g})$ in SDS sample buffer were subjected to $10 \%$ sodium dodecyl sulfate polyacrylamide gel electrophoresis (SDS-PAGE) and then electrophoretically transferred to immunoblotting polyvinylidene difluoride (PVDF) membranes. The membranes were treated with blocking solution (Thermo Fisher Scientific) at room temperature for $2 \mathrm{~h}$, and then the membranes were incubated with primary antibodies overnight at $4^{\circ} \mathrm{C}$. The following primary antibodies were used: mouse anti-APP polyclonal antibody (1:1000; Sigma), mouse anti- $\beta$-CTF polyclonal antibody (1:1000; Sigma), rabbit anti-BACE1 polyclonal antibody (1:1000; Abcam), mouse anti-NEP monoclonal antibody (1:1000; Abcam), rabbit anti-IDE polyclonal antibody (1:1000; Abcam), mouse anti-phospho-Tau (Ser202, Thr205) monoclonal antibody (AT8, 1:1000; Invitrogen), rabbit anti-phospho-tau (S396) monoclonal antibody (1:5000; Abcam), mouse anti-tau5 monoclonal antibody (1:1000; Abcam), rabbit anti-GSK-3 $\beta$ monoclonal antibody (1:1000; Cell Signaling Technology), rabbit anti-phospho-GSK3 $\beta$ monoclonal antibody (Ser9; 1:1000; Cell Signaling Technology), and mouse anti-beta actin monoclonal antibody (1:5000; Abcam). After washing in TBST, the membranes were incubated with goat antirabbit IgG $(\mathrm{H}+\mathrm{L})-\mathrm{HRP}$ conjugate (1:3000; Bio-Rad) and goat anti-mouse IgG (H+L)-HRP conjugate (1:3000; Bio-Rad) for $1 \mathrm{~h}$ at room temperature. After washing again in TBST, immunoreactive bands were detected by chemiluminescence reagents (ECL, Bio-Rad). Images of the protein bands were acquired using a Tanon 4500 system. ImageJ was utilized to scan the pixel density of the protein bands of the resultant blots.

\section{Quantitative Real-Time PCR}

Total RNA was extracted from hippocampal and cortical tissues using a total RNA kit (QIAGEN) according to the manufacturer's instructions. Reverse transcription was performed using a PrimeScript ${ }^{\mathrm{TM}}$ RT reagent kit (Takara), and real-time PCR was performed using a SYBR ${ }^{\circledR}$ Premix Ex Taq ${ }^{\mathrm{TM}}$ (Tli RNaseH Plus) kit (Takara) on an Applied Biosystems ${ }^{\circledR} 7500$ Fast Real-Time PCR Systems (Thermo Fisher Scientific). The $\triangle \triangle \mathrm{CT}$ method was used to calculate the fold changes in gene expression level between target genes and GAPDH. The primers used in the experiments are listed in Table $\mathbf{1 .}$

\section{A $\beta$ ELISA}

$\mathrm{A} \beta$ ELISA was performed as previously described [23, 24] (Liu et al., 2014; Fang et al., 2016). Briefly, cortical and 
TABLE 1 | Primers used for real-time PCR.

\begin{tabular}{|c|c|c|}
\hline Gene & Forward $\left(5^{\prime}-3^{\prime}\right)$ & Reverse $\left(5^{\prime}-3^{\prime}\right)$ \\
\hline IL-1 $1 \beta$ & GCCCATCCTCTGTGACTCAT & AGGCCACAGGTATITGTCG \\
\hline TNF- $\alpha$ & CCACCACGCTCTTCTGTCTAC & TGGGCTACAGGCTTGTCACT \\
\hline iNOS & ACCTTGTTCAGCTACGCCTT & САTTCCCAAATGTGCTTGTC \\
\hline COX-2 & ATGAGC ACAGGATTTGACCA & TGGGCTTCAGCAGTAATITG \\
\hline IL-6 & ACAAGTCGGAGGCTT & TTGCCATTGCACAACTCTT \\
\hline IL-4 & ATCATCGGCATTITGAACGAGG & TGCAGCTCCATGAGAACACTA \\
\hline Fizz1 & CTGCTACTGGGTGTGCTTGT & GGCAGTTGCAAGTATCTCCA \\
\hline YM1 & TCTATGCCTTTGCTGGAATG & CAGGTCCAAACTTCCATCCT \\
\hline $\operatorname{Arg} 1$ & CTCCAAGCCAAAGTCCTTAGAG & GGAGCTGTCATTAGGGACATC \\
\hline NEP & стCTCTGTGCTTGTCTTGCTC & GACGTTGCGTTCAACCAGC \\
\hline IDE & GAAGACAAACGGGAATACCGTG & CCGCTGAGGACTTGTCTGTG \\
\hline GAPDH & CTCCACTCACGGCAAATTCA & GCCTCACCCCATTTGATGTT \\
\hline
\end{tabular}

hippocampal tissues were homogenized in Tris-buffered saline (TBS) containing a protease inhibitor cocktail (Sigma) and then centrifuged at $16,000 \times g$ for $30 \mathrm{~min}$ at $4^{\circ} \mathrm{C}$. The supernatant (TBS-soluble fraction) was collected, and the pellets were homogenized in TBS supplemented with $1 \%$ Triton X-100 (TBST) containing a protease inhibitor cocktail (Sigma), sonicated for $5 \mathrm{~min}$ at $4^{\circ} \mathrm{C}$ in a water bath, and then centrifuged at $16,000 \times g$ for another $30 \mathrm{~min}$ at $4^{\circ} \mathrm{C}$. The supernatant (TBST-soluble fraction) was collected, and the pellets were extracted a third time with an icecold guanidine buffer (5 $\mathrm{M}$ guanidine $\mathrm{HCl} / 50 \mathrm{mM}$ Tris, $\mathrm{pH}$ 8.0) and referred to as the guanidine-soluble fraction. The protein concentration was determined using a bicinchoninic acid protein assay kit (Beyotime Biotechnology). The A $\beta$ concentrations were detected by $\mathrm{A} \beta 40$ and $\mathrm{A} \beta 42$ ELISA kits (Invitrogen) following the manufacturer's instructions. The levels of $A \beta$ were normalized to the amount of total protein in the tissues.

\section{Statistical Analysis}

Data are reported as the mean \pm standard deviation (SD). Student's $t$-test was used to compare the two groups. One-way analysis of variance (ANOVA) followed by post hoc Tukey's test was used for multigroup comparisons. Statistical analyses were performed using GraphPad Prism 5 software (GraphPad Software). Values of $p<0.05$ were considered statistically significant.

\section{RESULTS}

\section{Characterization of the MenSCs}

The morphology and immunophenotyping of MenSCs were similar to MSCs. As shown in Figure 1, MenSCs were spindleshaped and plastic adherent cells (Figure 1A) that were positive for CD29, CD73, CD90, and CD105, which are markers of MSCs, and negative for CD34, CD117, and HLA-DR (Figure 1B). The results indicated that MenSCs were not haematopoietic and had low immunogenicity.

\section{MenSC Transplantation Ameliorates Spatial Learning and Memory Impairments in APP/PS1 Mice}

To estimate whether MenSC transplantation could improve cognition function and memory deficits of PBS-treated mice, MenSC-treated APP/PS1 mice and WT littermates, we used the Morris water maze to evaluate the spatial learning 21 days after MenSC transplantation. The Morris water maze is hippocampus dependent and can be used to evaluate the cognitive function of APP/PS1 mice. As presented in Figure 2, the PBS-treated APP/PS1 mice showed a significantly longer escape latency compared with their WT littermates, whereas the spatial learning and memory of APP/PS1 mice was rescued by treatment with MenSCs. APP/PS1 mice treated with MenSCs exhibited a significantly shorter escape latency than the mice treated with PBS (Figure 2A). Furthermore, the swimming paths of the WT and MenSC-treated APP/PS1 mice were target-oriented, while the PBS-treated APP/PS1 mice swam in circles to find the platform with no target orientation (Figure 2C). In the probe test, the platform was removed to evaluate the number of times each mouse crossed the original location of the platform in $60 \mathrm{~s}$. The MenSC transplantation mice crossed the original location of the platform more often than the PBS-treated APP/PS1 mice (Figure 2B). These results indicated that MenSC transplantation could ameliorate learning and memory impairments in APP/PS1 mice.

\section{MenSC Transplantation Reduces A $\beta$ Plaque Deposition and Levels in APP/PS1 Mice}

To assess the effects of MenSC transplantation on $\mathrm{A} \beta$ deposition, MenSCs were transplanted into the hippocampus of APP/PS1 mice at 7 months old, and the mice were subsequently sacrificed at 8 months old. The brain sections were stained with thioflavin $\mathrm{S}$ and $6 \mathrm{E} 10$ (A $\beta$ antibody) to evaluate $A \beta$ plaque deposition. Compared with PBS-treated mice, less $\mathrm{A} \beta$ plaques were observed in the hippocampus and the cortex of the MenSC-treated APP/PS1 mice as determined by thioflavin S staining (Figures 3C,D). Consistent with thioflavin $\mathrm{S}$ staining, quantitative analysis showed that 6E10-positive areas were significantly lower in the MenSCtransplanted APP/PS1 mice than in the PBS-treated mice (Figures 3A,B).

To further investigate the effect of MenSCs on $A \beta$ pathology, the levels of different $A \beta$ isoforms, $A \beta 40$ and $A \beta 42$, were measured in the hippocampal and cortical tissues by using ELISA. $\mathrm{A} \beta 40$ and $\mathrm{A} \beta 42$ are the most toxic $A \beta$ isoforms and are enriched in monomers, oligomers and $\mathrm{A} \beta$ plaques in the hippocampus and cortex. The results revealed that MenSC administration markedly decreased the concentrations of A $\beta 40$ and A $\beta 42$ in TBS-, TBST, and guanidine chloride-fractions in both the hippocampus and cortex in comparison with PBS administration (Figures 3E,F).

$\mathrm{A} \beta$ is a proteolytic product of APP. To examine whether the reduction of $A \beta$ deposition after MenSC transplantation was associated with the metabolic processing of APP, we used 
A

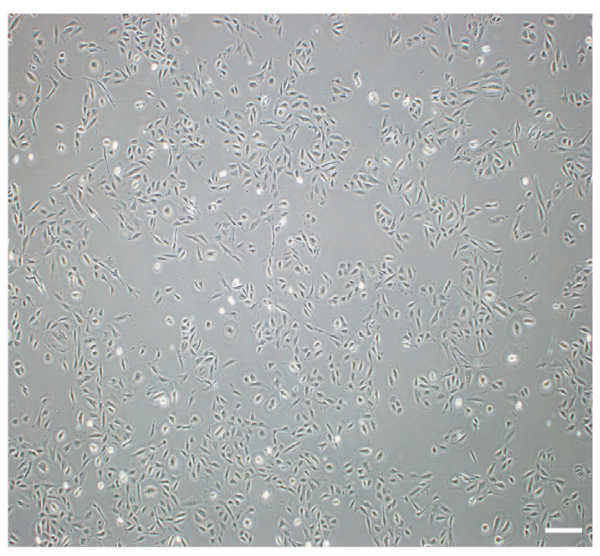

C
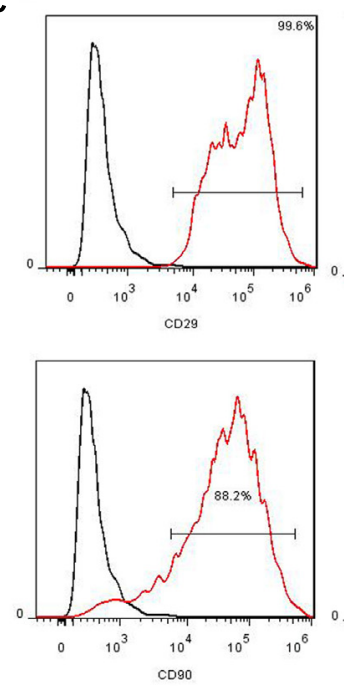

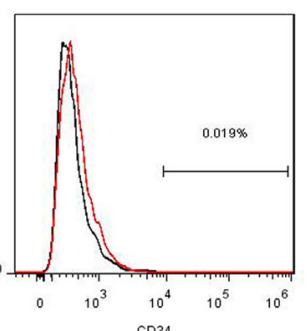

CD34

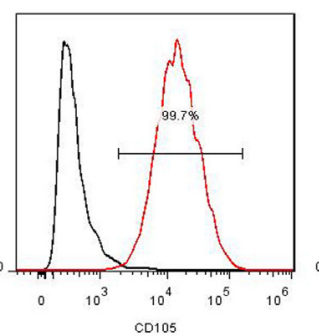

B
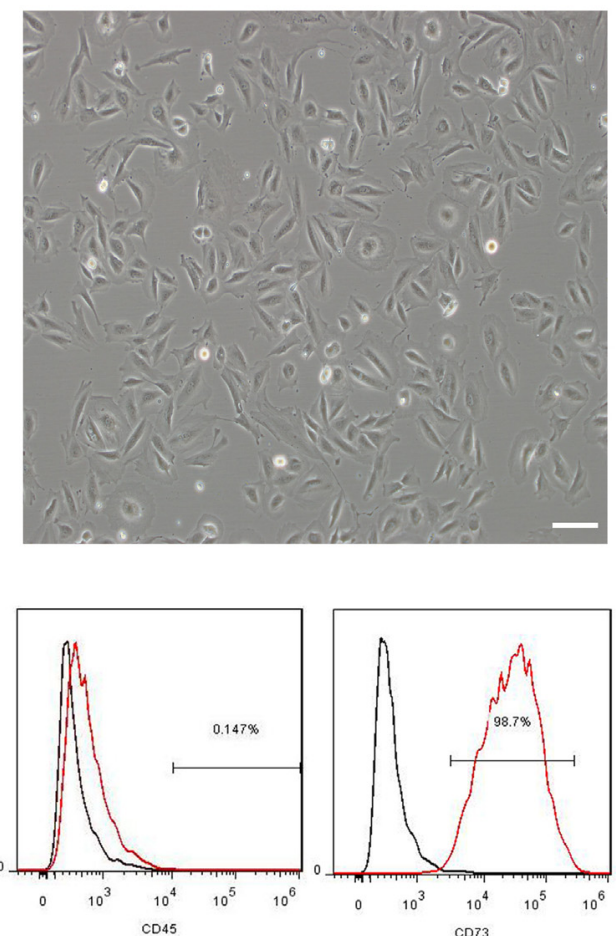

CD45

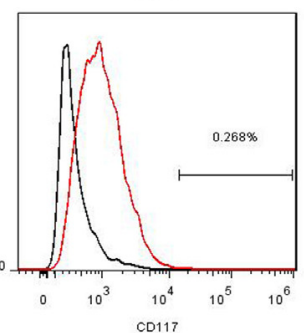

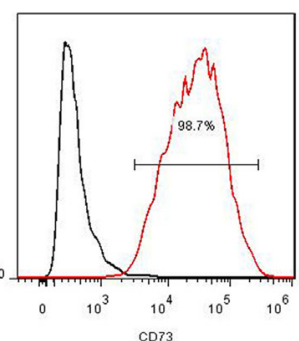

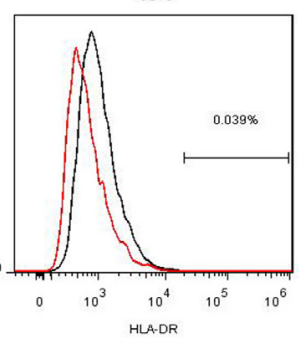

FIGURE 1 | Characterization of MenSCs. (A,B) Representative images of MenSCs. Scale bars $=200 \mu \mathrm{m}$ (A) and $100 \mu \mathrm{m}$ (B). (C) Flow cytometry analysis of the surface markers of MenSCs. The black lines represent the isotype control, and the red lines represent the level of surface markers. MenSCs, human menstrual blood-derived stem cells.

Western blot to investigate the expression of APP in the hippocampal and cortical tissues of APP/PS1 mice. Western blot analysis revealed that there were no significant differences in APP expression between APP/PS1 mice treated with MenSCs or PBS (Figures 4A,B), indicating that the level of APP expression was not changed after transplantation of MenSCs in the hippocampus and cortex of APP/PS1 mice. In the amyloidogenic pathway, APP is cleaved by $\beta$-secretase (BACE1) to generate a C-terminal fragment $(\beta-\mathrm{CTF})$, and then $\beta$-CTF is further cleaved by $\gamma$-secretase to produce $A \beta$ peptides. Therefore, we examined BACE1 by Western blot, and the results revealed that MenSC transplantation markedly decreased the levels of BACE1 in the brains of APP/PS1 mice (Figures 4E,F). Furthermore, western blot analysis of $\beta$-CTF suggested that $\beta$-CTF had declined in the MenSC-treated APP/PS1 mice (Figures 4C,D). Taken together, MenSC transplantation reduced $A \beta$ deposition in the brains of APP/PS1 mice, probably by inhibiting the activity of $\beta$-secretase.

\section{MenSC Transplantation Stimulates Microglial Activation in the Brains of APP/PS1 Mice}

In previous studies, our group proved that MenSCs could alleviate the inflammatory response in several diseases (Chen et al., 2017a; Xiang et al., 2017). Microglia are generally considered the immune cells in the CNS and are frequently associated with $\mathrm{A} \beta$ plaques in $\mathrm{AD}$ brains. Recent reports also have suggested that MSCs increase microglial activation in APP/PS1 mice (Lee et al., 2010; Lee H.J. et al., 2012). Therefore, we further evaluated whether MenSC transplantation could affect microglial activity in APP/PS1 mice. Mouse brain sections were stained with Iba-1, which is a marker of activated microglia. The results revealed that Iba-1-positive cells were widely distributed and markedly increased in the hippocampus and cortex of the MSC-treated APP/PS1 mice compared with the PBS-treated mice (Figures 5A,C). Quantitative analysis revealed 
A

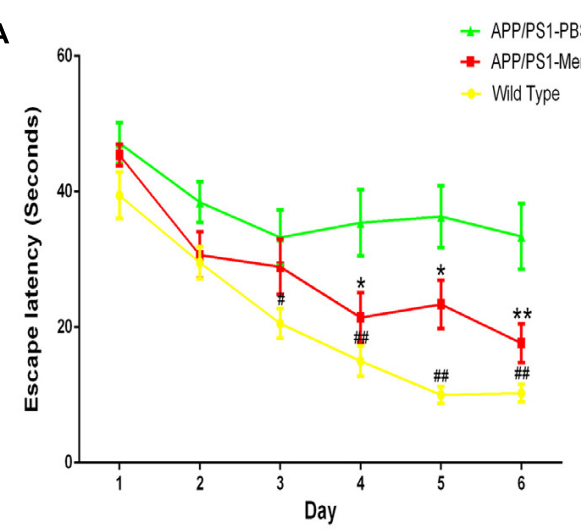

B

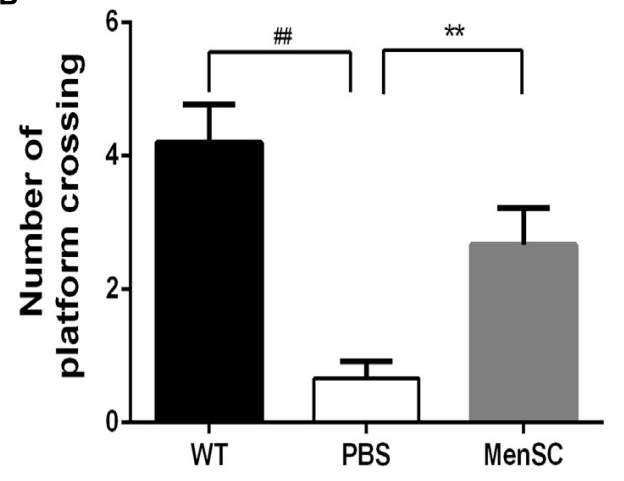

C

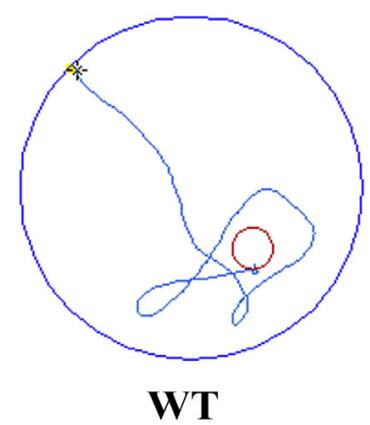

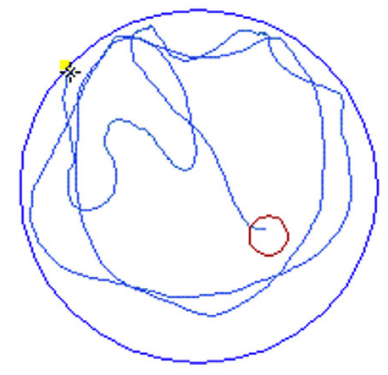

PBS

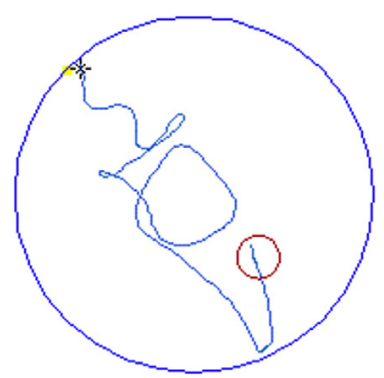

MenSC

FIGURE 2 | MenSC transplantation rescues spatial learning and memory impairments in APP/PS1 mice. (A) Escape latency to reach the hidden platform on 6 successive days in the Morris water maze for WT and APP/PS1 mice treated with MenSCs or PBS. For each day of training, the mean of 4 trials per day is presented. During training, MenSC-treated APP/PS1 mice required a shorter amount of time to reach the hidden platform than PBS-treated APP/PS1 mice. (B) The number of crossings of the location of the platform during the probe trial. The results represent the number of times each animal crossed the previous location of the platform within $1 \mathrm{~min}$, which was removed in the probe trials. (C) Representative swimming paths of one mouse per group on day 6 illustrating the escape latency in the Morris water maze. The red circle shows the location of the former platform. Values are represented as the means \pm SEM. Significant differences between PBS-treated and MenSC-treated APP/PS1 mice: ${ }^{*} p<0.05$ and ${ }^{* *} p<0.01$, WT and PBS-treated APP/PS1 mice: $\#<0.05$ and ${ }^{\# \#} p<0.01, n=15$ per group. MenSCs, human menstrual blood-derived stem cells.

that the MenSC-treated APP/PS1 mice displayed a greater area of Iba-1 immunoreactivity than the PBS-treated mice in both regions of the hippocampus and cortex (Figures 5B,D). Collectively, these results suggested that the activation of microglia could be improved after transplanting MenSCs into APP/PS1 mice.

\section{MenSC Transplantation Induces Activated Microglia Toward an Alternative Phenotype and Enhances A $\beta$-Degrading Enzyme Activity}

Microglia are frequently related to $\mathrm{A} \beta$ plaques in $\mathrm{AD}$ brains. In previous experiments, we demonstrated that MenSC transplantation promoted the activation of microglia. It has also been reported that activated microglia can be either detrimental or beneficial in neuroinflammation. For example, microglia can be activated by $A \beta$ deposition and increase the expression of proinflammatory cytokines, such as tumour necrosis factor (TNF) $-\alpha$ and interleukin (IL)-1 $\beta$. Therefore, we quantified the mRNA expression of several proinflammatory factors and reactive oxygen species to investigate whether activated microglia aggravated neuroinflammation in APP/PS1 mice. Compared with the PBS-treated APP/PS1 mice, the expression of these cytokines was dramatically decreased in both the hippocampus and cortex of the MenSC-treated APP/PS1 mice. Interestingly, MenSCs administration stimulated microglial activation, yet the expression of proinflammatory cytokines decreased (Figures 5E,F). As microglial activation phenotypes are dynamic, we questioned whether MenSCs induced activated microglia to exhibit an alternative phenotype that exerts protective effects and reduces proinflammation cytokines to prevent neurotoxicity. IL-4, YM-1, Arg-1, Fizz1, and CD206 are associated with and strong expressed in alternative activation of microglia. Quantitative real-time PCR showed that the mRNA expression of IL-4, YM-1, Arg-1, Fizz1, and CD206 cytokines was markedly upregulated in MenSCs in both the hippocampus and the cortex of APP/PS1 mice (Figures 5G,H). Therefore, we considered that the activated microglia convert to an alternative phenotype, which is beneficial in reducing $A \beta$ plaques. Next, colocalization of microglia with A $\beta$ plaques in the brains of APP/PS1 mice was determined 
A

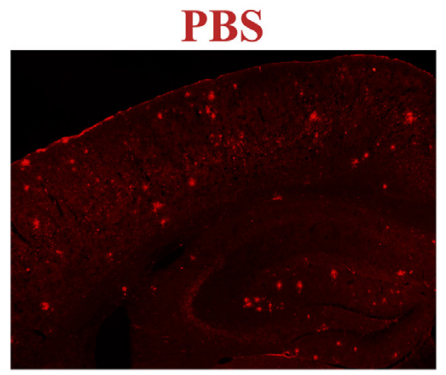

C

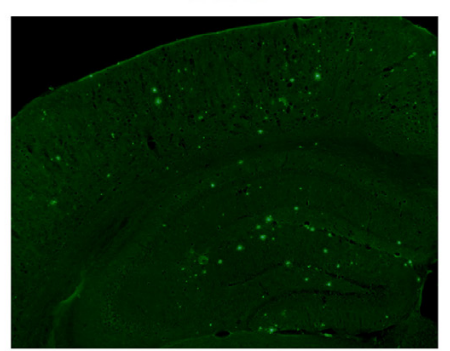

E

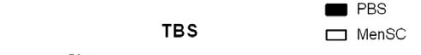

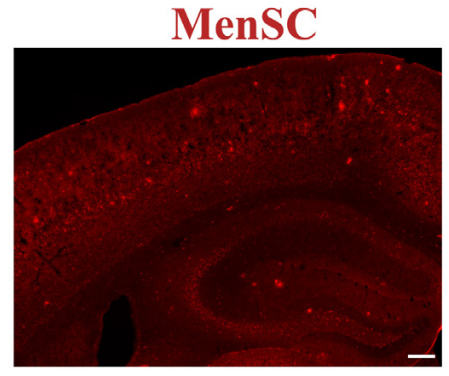

MenSC

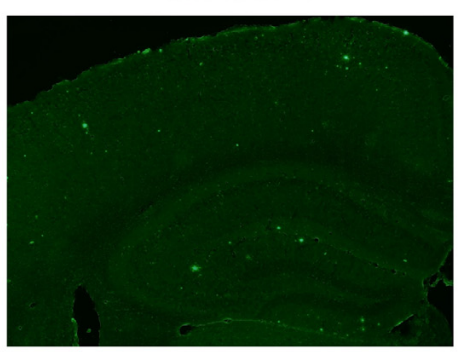

B

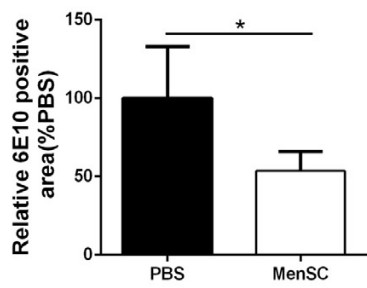

D

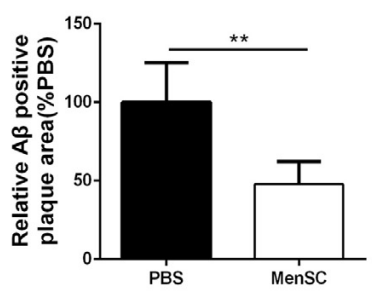

GuHCL

口 MBS
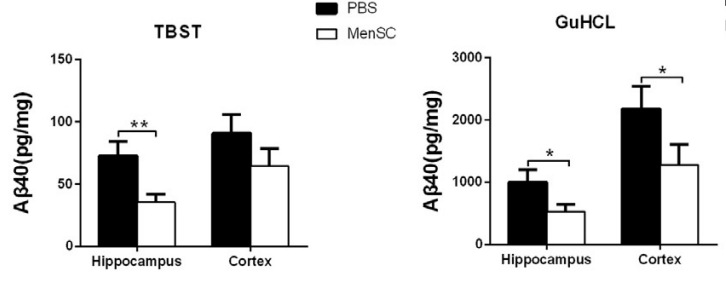

$\begin{array}{ll}\text { TBST } & \square \text { PBS }\end{array}$

GuHCL

PBS

$\mathbf{F}$
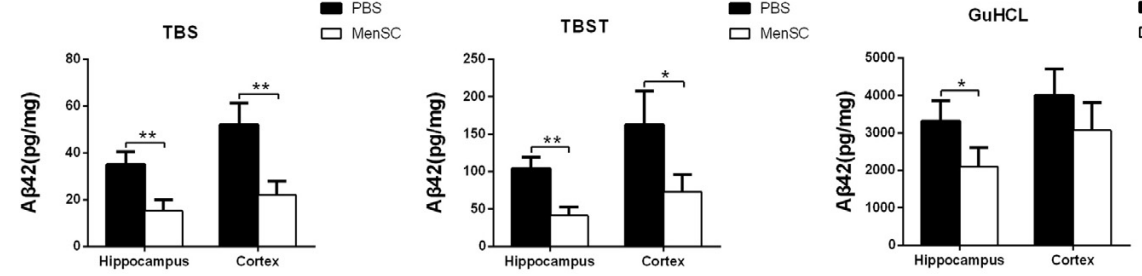

FIGURE 3 | MenSC transplantation reduced AB deposition in APP/PS1 mice. (A) $6 E 10$ antibody-stained brain sections of APP/PS1 mice treated with MenSCs compared with those of APP/PS1 mice treated with PBS. (B) Quantification of the 6E10-positive fraction showed a significant reduction in the hippocampus and the cortex of MenSC-treated APP/PS1 mice. Values are represented as a percentage of control (PBS-treated APP/PS1 mice). Scale bar $=200 \mu \mathrm{m}, n=5$ per group. (C) Brain sections stained with thioflavin S in APP/PS1 mice treated with MenSCs or PBS. (D) Quantification of the thioflavin S-positive area demonstrated a marked decline in both the hippocampus and the cortex of APP/PS1 mice treated with MenSCs. Values are represented as a percentage of control (PBS-treated APP/PS1 mice). Scale bar $=200 \mu \mathrm{m}, n=5$ per group. (E,F) Hippocampal and cortical tissues of APP/PS1 mice were homogenized and separated into TBS, TBST, and guanidine fractions. The levels of $A \beta 40$ and $A \beta 42$ were measured by using ELISA. Reductions in $A \beta 40$ and $A \beta 42$ were observed in the hippocampal and cortical samples of APP/PS1 mice treated with MenSCs. Values are represented as the means \pm SD. Significant differences between PBS-treated and MenSC-treated APP/PS1 mice: ${ }^{*} p<0.05$ and ${ }^{* *} p<0.01, n=3$ per group. MenSCs, human menstrual blood-derived stem cells; A $\beta$, amyloid beta.

by immunofluorescence double staining of Iba-1 and $6 \mathrm{E} 10$ to further clarify the relationship between microglial activation and the reduction in $A \beta$ deposition following transplantation of MenSCs. We noted that more Iba-1-positive cells were clustered adjacent to the center of $A \beta$ plaques in MenSCtreated APP/PS1 mice, whereas Iba-1-positive cells tended to distribute around the $\mathrm{A} \beta$ plaques in PBS-treated APP/PS1 mice (Figure 6A). Alternatively activated microglia can secrete $\mathrm{A} \beta$-degrading enzymes, including insulin-degrading enzyme (IDE) and neprilysin (NEP), which are two major proteases
(Iwata et al., 2001; Farris et al., 2003). Therefore, we measured the mRNA expression of IDE and NEP in the hippocampus and cortex of APP/PS1 mice. The results showed that MenSC transplantation dramatically increased IDE and NEP mRNA expression in APP/PS1 mice (Figures 6B,C). Moreover, western blot analysis revealed that MenSC transplantation markedly increased the levels of NEP and IDE in the brains of APP/PS1 mice (Figures 6D-I). These results suggested that MenSC transplantation induced the activated microglia to display the alternative phenotype and enhanced $A \beta$-degrading enzyme 
A

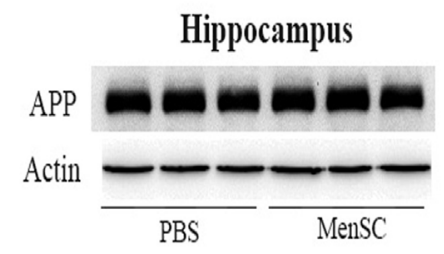

C

E

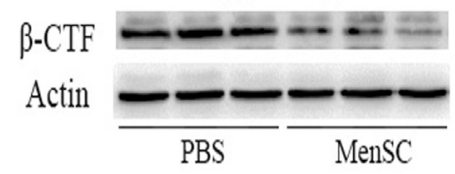

Hippocampus

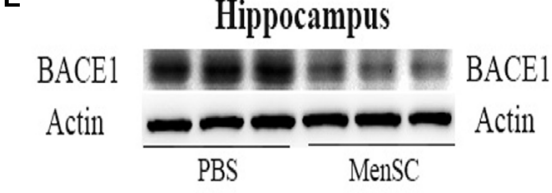

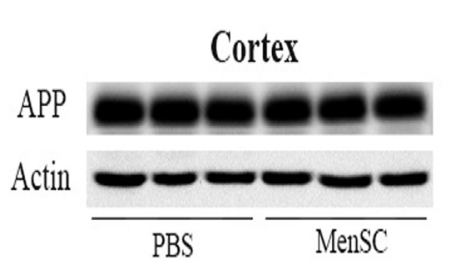

B
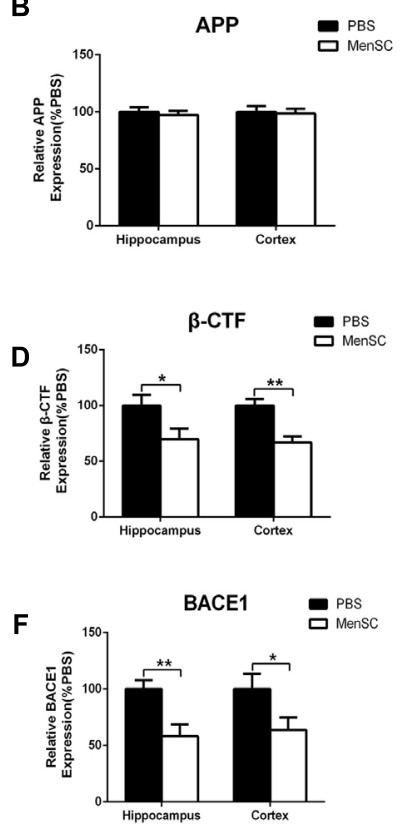

FIGURE 4 | MenSC transplantation decreased BACE1 and $\beta$-CTF expression in the hippocampus and cortex of APP/PS1 mice. (A,C,E) Western blot analysis of APP, $\beta$-CTF, and BACE1 in hippocampal and cortical areas of APP/PS1 mice treated with MenSCs or PBS. (B,D,F) Quantification of Western blots in (A,C,E), respectively, show protein as a percentage of control (PBS-treated APP/PS1 mice) normalized to actin. Values are represented as the means \pm SD. Significant differences between PBS-treated and MenSC-treated APP/PS1 mice: ${ }^{*} p<0.05$ and ${ }^{* *} p<0.01, n=3$ per group. MenSCs, human menstrual blood-derived stem cells; APP, amyloid precursor protein.

activity in APP/PS1 mice, which both helped to reduce $\mathrm{A} \beta$ deposition.

\section{MenSC Transplantation Decreases Tau Hyperphosphorylation in APP/PS1 Mice}

Neurofibrillary tangles composed of hyperphosphorylated tau protein are another pivotal pathological characteristic of AD. To assess whether MenSCs could affect tau pathology, tau phosphorylation at Ser202/Thr205 (AT8) and Ser396 sites was measured by Western blot. Western blot analysis showed that tau phosphorylation at Ser202/Thr205 (AT8) and Ser396 sites was dramatically downregulated in both the hippocampus and the cortex of MenSC-treated mice compared with PBS-treated mice, (Figures 7A-D). Next, to elucidate the mechanism through which MenSCs ameliorate tau phosphorylation in APP/PS1

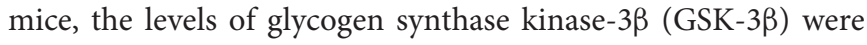
examined by using Western blot. GSK-3 $\beta$ is a serine/threonine protein kinase and is considered an important tau kinase. The expression of GSK-3 $\beta$ leads to tau phosphorylation at many sites, and GSK-3 $\beta$ phosphorylation at Ser9 leads to inhibition of its kinase activity (Llorens-Martín et al., 2014). Western blot analysis revealed that MenSC transplantation significantly increased the level of phosphorylated GSK-3 $\beta$ (Ser9) in the hippocampus and the cortex of APP/PS1 mice (Figures 7E,F). These results demonstrated that MenSC transplantation could reduce tau hyperphosphorylation by inactivating GSK-3 $\beta$.

\section{DISCUSSION}

Human menstrual blood-derived stem cells isolated from menstrual fluids are a novel source of MSCs (Figure 1) and have important advantages such as the ability to perform repeated, simple sampling and to circumvent ethical issues compared with MSCs derived from other sources. Moreover, MenSCs can be periodically isolated from donors because the isolation procedure is non-invasive, thus ensuring higher therapeutic doses with fewer cell passages (Khoury et al., 2014; Alcayaga-Miranda et al., 2015). These characteristics make MenSCs an ideal candidate for $\mathrm{AD}$ therapy. In the current study, the effects of intracerebral MenSC transplantation on $\mathrm{A} \beta$ deposition, tau pathology and cognitive decline were evaluated in APP/PS1 double-transgenic mice for the first time. Our results show that MenSC transplantation reduced $\mathrm{A} \beta$ deposition (Figure 3), which was associated with an alleviation of abnormal APP processing (Figure 4) and restoration of $A \beta$ clearance by microglia (Figures 6B,C), and decreased tau hyperphosphorylation, which was led to inactive GSK-3 $\beta$ (Figure 7), in APP/PS1 mice. Both the hippocampus and the cortex benefitted from MenSC transplantation. Concomitantly, MenSC transplantation stimulated microglial activation and induced activated microglia (Figures 5A-D) to express the alternative phenotype characterized by the secretion of anti-inflammatory cytokines (Figures 5E-H) rather than the neurotoxic phenotype. More importantly, MenSCs protected APP/PS1 mice 


\section{A}

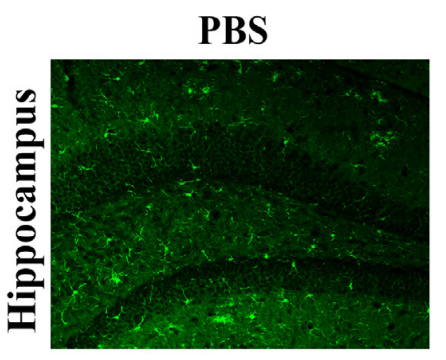

C

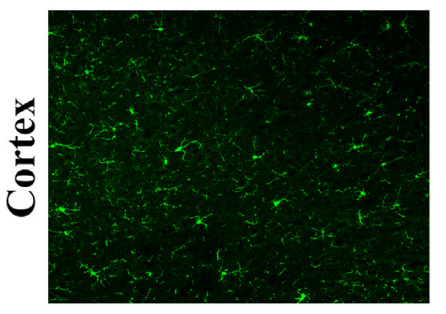

E
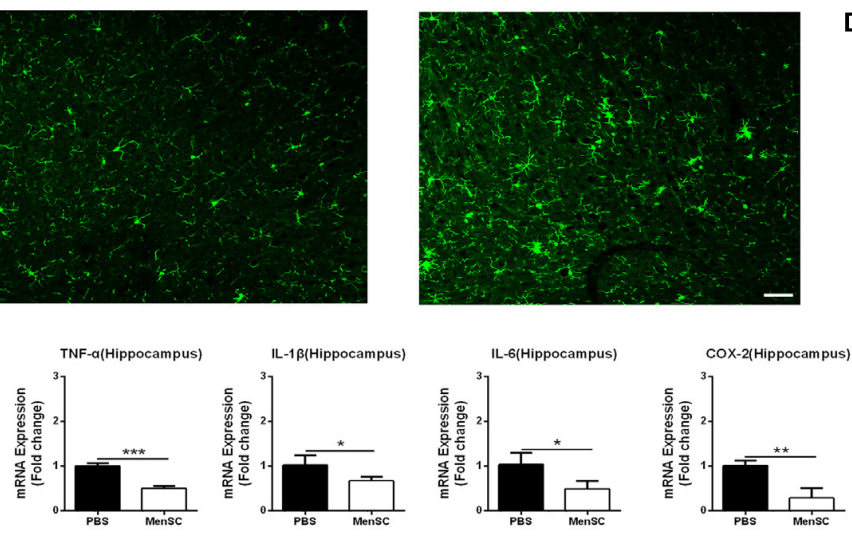

D
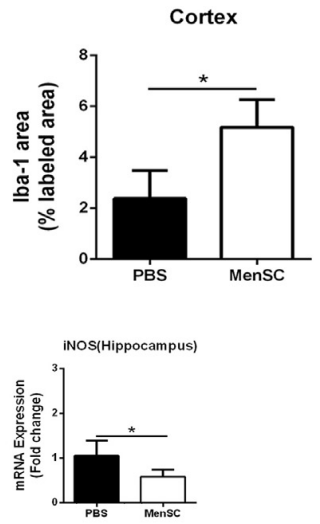

$\mathbf{F}$

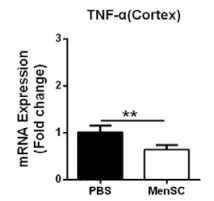

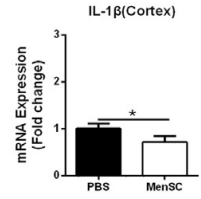
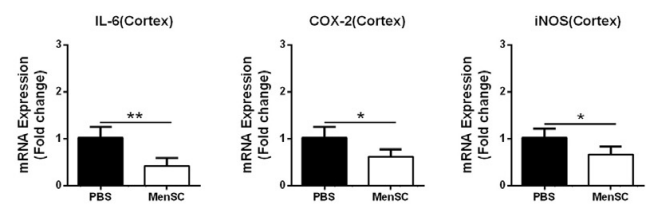

G
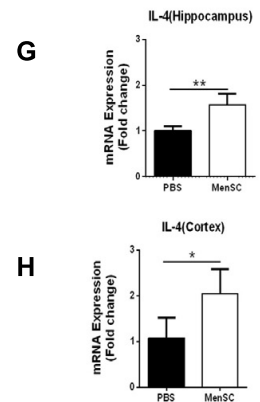
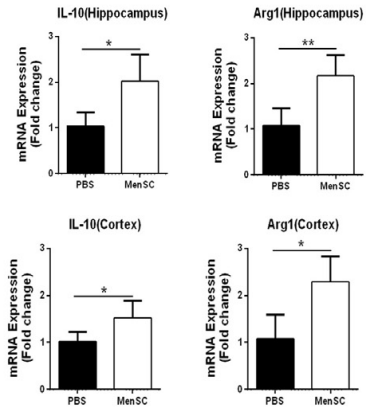
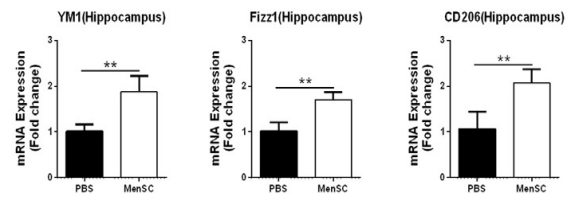

FIGURE 5 | MenSC transplantation stimulated microglial activation and induced activated microglia toward the alternative phenotype. (A,C) Immunofluorescent staining for lba-1 in brain sections of APP/PS1 mice treated with PBS or MenSCs. Scale bar = $100 \mu \mathrm{m}, n=5$ per group. (B,D) Quantification of the percentages of the hippocampus and cortex exhibiting Iba-1 immunoreactivity. (E,F) Expression of TNF- $\alpha, \mathrm{IL}-1 \beta$, IL-6, COX-2, and iNOS was measured by quantitative real-time PCR in the hippocampus and the cortex of APP/PS1 mice treated with PBS or MenSCs ( $n=4$ per group). The expression of these cytokines was significantly decreased in APP/PS1 mice after MenSC treatment. (G,H) The expression of IL-4, YM1, Arg1, CD206, IL-10, and Fizz1, which are markers of alternatively activated microglia, was measured by quantitative real-time PCR in the hippocampus and the cortex of APP/PS1 mice treated with PBS or MenSCs ( $n=4$ per group). There were significant increases in the mRNA expression of these markers in MenSC-treated APP/PS1 mice compared with APP/PS1 mice treated with PBS. Values are represented as the means \pm SD. Significant differences between PBS-treated and MenSC-treated APP/PS1 mice: * $p<0.05$ and ** $p<0.01$. MenSCs, human menstrual blood-derived stem cells.

against cognitive decline and memory deficits (Figure 2). Taken together, the treatment effectively attenuated $\mathrm{AD}$ pathology by targeting multiple key pathways in APP/PS1 mice.

Analysis of $6 \mathrm{E} 10$ and thioflavin $S$ staining revealed that $\mathrm{A} \beta$ deposition in the hippocampus and cortex of MenSC-treated
APP/PS1 mice was significantly reduced (Figures 3A-D), and the ELISA results for $A \beta 40$ and $A \beta 42$ were consistent with $6 \mathrm{E} 10$ and thioflavin $S$ staining (Figures 3E,F). In the amyloidogenic pathway, APP is cleaved by $\beta$-secretase (BACE1) to generate a $\mathrm{C}$-terminal fragment ( $\beta$-CTF), and then $\beta$-CTF is further cleaved by $\gamma$-secretase to produce $A \beta$ peptides (Zhang et al., 2011). 
A
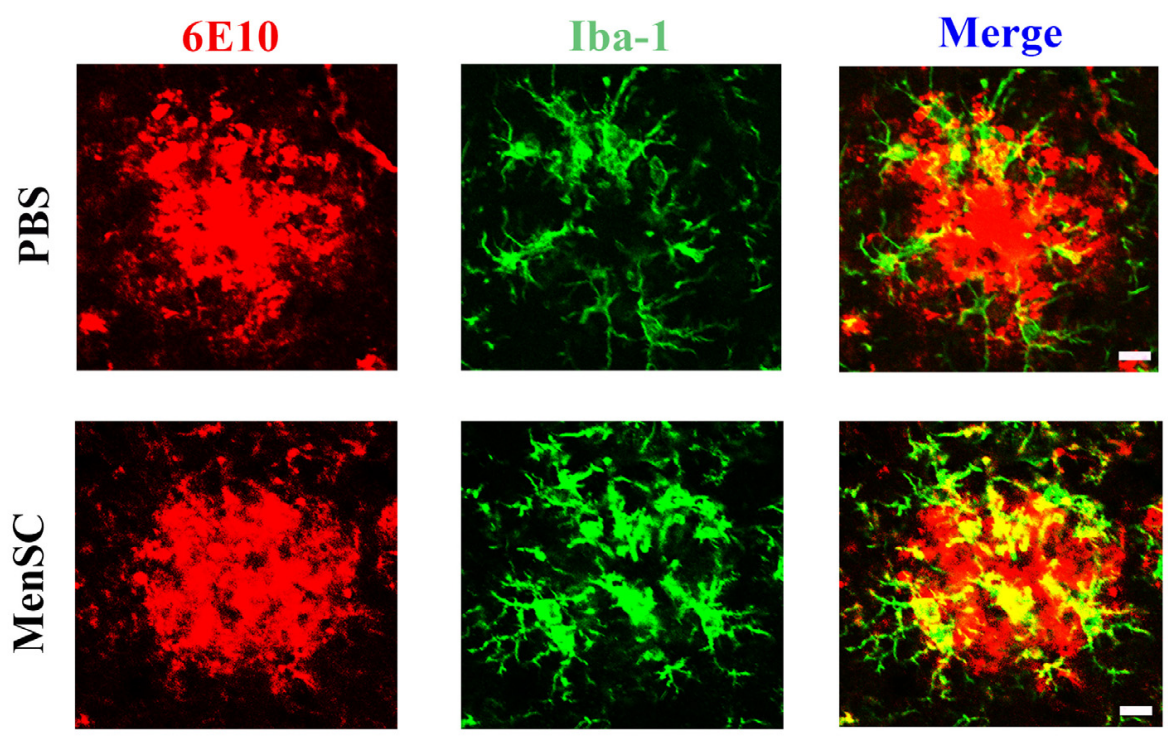

B

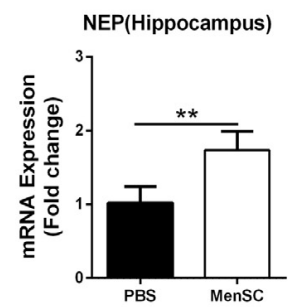

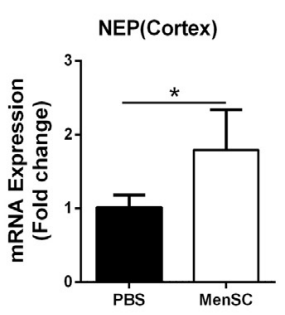

C
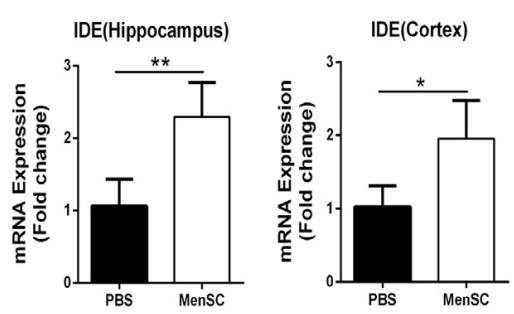

D

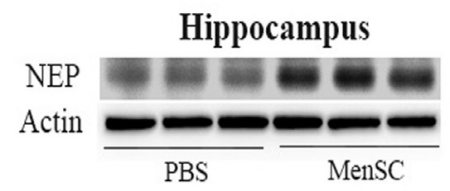

G

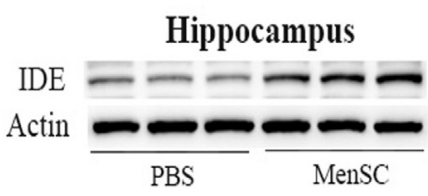

E
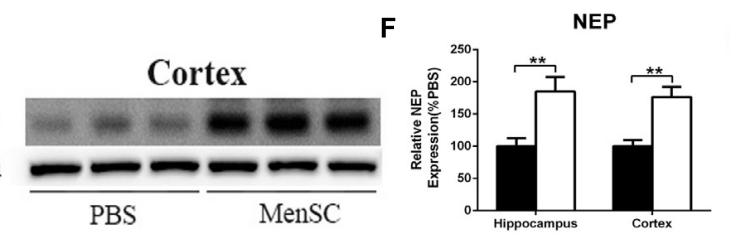

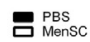

H

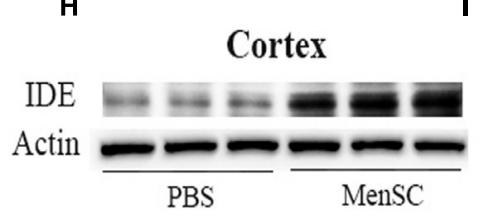

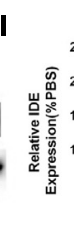

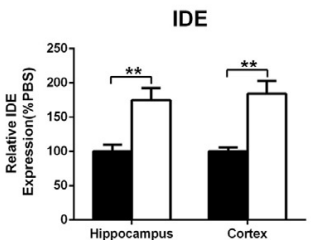

FIGURE 6 | Transplanted MenSCs recruited microglia to cluster adjacent to the center of A $\beta$ plaques and restored microglial A $\beta$ clearance capacity in APP/PS1 mice. (A) Confocal microscopy was used to assess Iba-1 colocalization with 6E10 in APP/PS1 mice treated with MenSCs or PBS. Merged images of Iba-1 and 6E10 showed that more microglia clustered adjacent to the center of A $\beta$ plaques in the brains of MenSC-treated APP/PS1 mice. Scale bar = 20 $\mu \mathrm{m}$. (B,C) The expression of A $\beta$ degrading enzymes NEP and IDE in the hippocampus and the cortex of APP/PS1 mice treated with PBS or MenSCs $(n=4)$. (D,E,G,H) Western blot analysis of NEP and IDE in hippocampal and cortical areas of APP/PS1 mice treated with MenSCs or PBS. (F,I) Western blots in (D,E,G,H), respectively, show protein as a percentage of control (PBS-treated APP/PS1 mice) normalized to actin. Values are represented as the means \pm SD. Significant differences between PBS-treated and MenSC-treated APP/PS1 mice: * $p<0.05$ and ${ }^{* *} p<0.01$. MenSCs, human menstrual blood-derived stem cells; IDE, insulin-degrading enzyme; NEP, neprilysin (NEP).

During this process, BACE1 is considered the initial and ratelimiting enzyme (Ye and Cai, 2014). Therefore, many studies have focused on elucidating the role of BACE1 in AD. It has been reported that BACE1 knockout $\left(\mathrm{BACE}^{-/-}\right)$prevents the development of $\mathrm{AD}$ pathologies and cognitive decline in mouse models of AD (Ohno et al., 2004). Moreover, BACE1 activity increases with age and is increased in the brains of $\mathrm{AD}$ patients (Yang et al., 2003; Fukumoto et al., 2004). Here, we observed that MenSC transplantation decreased BACE1 and $\beta$-CTF levels in the hippocampus and cortex of APP/PS1 mice (Figure 4). According 


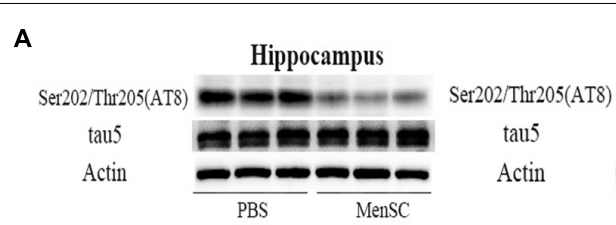

C

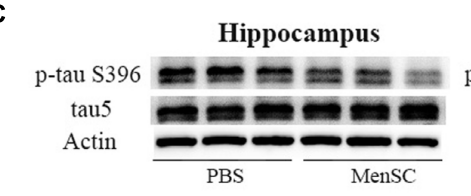

E

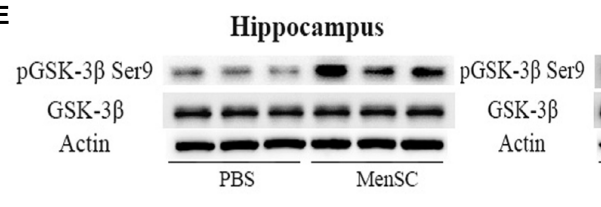

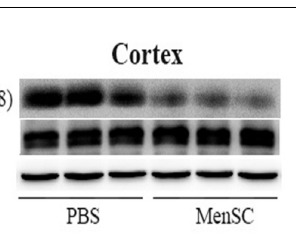

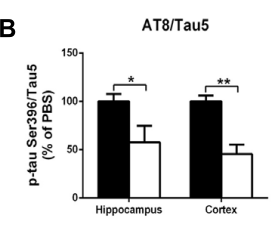

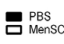

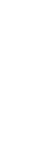

D

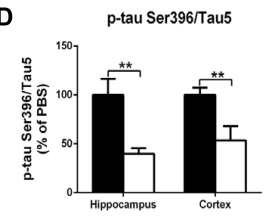

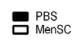
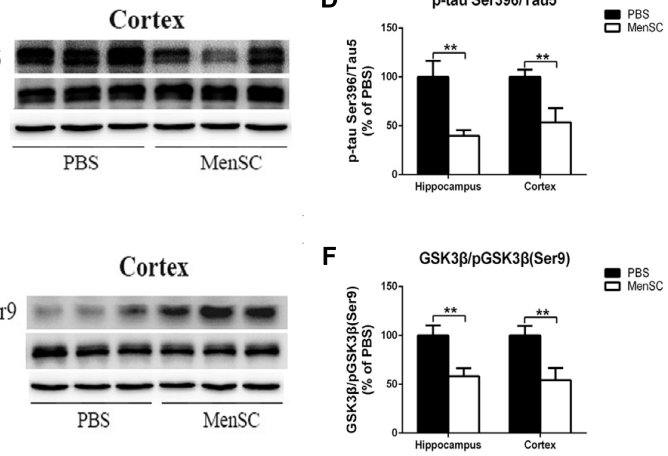

FIGURE 7 | MenSC transplantation ameliorates tau hyperphosphorylation in APP/PS1 mice. (A,C) Western blot analysis of tau phosphorylation at Ser202/Thr205 (AT8) and Ser396 sites in the hippocampus and the cortex of APP/PS1 mice treated with PBS or MenSCs. (B,D) Quantification of the Western blot results in (A,C) presented as the ratio of AT8/Tau5 and Ser396/Tau5 as a percentage of control (PBS-treated APP/PS1 mice). (E) Western blot analysis of GSK3 $\beta$ and phosphorylated GSK3 $\beta$ at the Ser9 site in the hippocampus and the cortex of APP/PS1 mice treated with PBS or MenSCs. (F) Quantification of the Western blot results in (E) presented as the ratio of GSK3 $\beta /$ pGSK3 $\beta$ (Ser9). Values are represented as the means \pm SD. Significant differences between PBS-treated and MenSC-treated APP/PS1 mice: ${ }^{*} p<0.05$ and ${ }^{* *} p<0.01, n=3$. MenSCs, human menstrual blood-derived stem cells; GSK3 $\beta$, Glycogen synthase kinase 3 beta.

to our results, we attribute the beneficial effect of MenSCs on reducing $\mathrm{A} \beta$ deposition, at least in part, to inhibiting $\mathrm{BACE} 1$ activity.

Microglia, the resident immune cells in the CNS, play a critical role in immune surveillance of the brain (Boche et al., 2013). Activated microglia exert both toxic and beneficial effects depending on their phenotype in $\mathrm{AD}$ progression. It is well established that $A \beta$ plaques sustain the activation of microglia, resulting in constant production of inflammatory cytokines and reactive oxygen species, which lead to neurodegeneration (Pimplikar, 2014; Ransohoff, 2016). On the other hand, alternatively activated microglia exert neuroprotective effects in $\mathrm{AD}$ that depend on attenuating inflammation and phagocytizing $\mathrm{A} \beta$, which are related to the increased secretion of neurotrophic factors and proteases (Heneka et al., 2014). Our current findings showed that MenSC transplantation stimulated microglial activation (Figures 5A-D). Next, real-time PCR was performed to examine the expression of IL-1 $\beta$ and TNF- $\alpha$ to investigate whether activated microglia stimulated by MenSCs produced proinflammatory cytokines. Strikingly, we observed that the expression of these proinflammatory factors was significantly decreased after MenSC transplantation in the brains of APP/PS1 mice (Figures 5E,F). It has been reported that MSC transplantation can induce activated microglia to express the alternative phenotype rather than the neurotoxic phenotype in APP/PS1 mice (Lee et al., 2010; Lee H.J. et al., 2012). Thus, we investigated whether MenSC transplantation induced activated microglia to display the alternative phenotype. It is widely acknowledged that IL-4 is a pivotal anti-inflammatory cytokine that enhances the alternative phenotype of activated microglia in vivo and improves $\mathrm{A} \beta$ removal (Heneka et al., 2014; Latta et al., 2015). YM1, Arg1, Fizz1 and CD206 which are recognized as markers of the alternative activation of macrophages, are also strongly expressed in alternatively activated microglia. It is compelling that MenSC transplantation dramatically increased the mRNA levels of IL-4, YM1, Arg1, Fizz1, and CD206 in both the hippocampus and the cortex of APP/PS1 mice (Figures $\mathbf{5 G}, \mathbf{H}$ ). These results clearly demonstrated that MenSC transplantation induced activated microglia toward the alternative phenotype, which exerted beneficial effects in AD.

Insulin-degrading enzyme and neprilysin are the major $\mathrm{A} \beta$-degrading enzymes and can be secreted by microglia in the brain (Tuppo and Arias, 2005). However, impaired and dysfunctional microglia decrease the capacity of $A \beta$ clearance in $\mathrm{AD}$ model mice due to reduced IDE and NEP secretion (Hickman et al., 2008). Recent studies have revealed that soluble intracellular adhesion molecule-1 (sICAM-1) secreted by human umbilical cord blood-derived MSCs decreased A $\beta$ plaques by inducing NEP expression in microglia in APP/PS1 mice (Kim et al., 2012), and soluble CCL5 derived from bone marrow-derived MSCs modulated the microglial activation status and reduced $A \beta$ deposition by expression of $A \beta$-degrading enzymes in APP/PS1 mice (Lee J.K. et al., 2012). Moreover, it has been reported that MSC transplantation can promote the alternative activation of microglia and enhance the secretion of NEP and IDE by alternatively activated microglia (Ma et al., 2013). In our study, we observed that activated microglia clustered adjacent to the center of $\mathrm{A} \beta$ plaques in response to MenSC transplantation, whereas activated microglia distributed around the $A \beta$ plaques in PBS-treated mice (Figure 6A). 
The quantitative real-time PCR and western blot analysis showed that MenSC transplantation significantly increased the expression of NEP and IDE in the hippocampus and the cortex of APP/PS1 mice (Figures 6B-I). Based on these results, we proposed that MenSC transplantation induced activated microglia to convert to the alternative phenotype and that the positive effects of MenSCs were conferred through upregulated levels of $\mathrm{A} \beta$-degrading enzymes, leading to increased $\mathrm{A} \beta$ clearance. The beneficial effect of MenSC transplantation on reducing $A \beta$ deposition was associated with inhibiting BACE1 activity and restoring $A \beta$-clearance via microglia. In addition to these two pathways, the influx and efflux of brain $\mathrm{A} \beta$ also mediate $\mathrm{A} \beta$ clearance, which may be another pathway that could improve $A \beta$ clearance and deserves further study.

Intracellular NFTs composed of phosphorylated microtubuleassociated tau protein is another pathological hallmark of $\mathrm{AD}$ (Ballard et al., 2011). Under abnormal phosphorylation, tau lessens its affinity and separates from microtubules, which damages microtubule function and affects axonal transport (Spillantini and Goedert, 2013). Among the main aberrantly hyperphosphorylated sites on tau, Ser202/Thr205 (AT8) and Ser396 are the pathological phosphorylated sites (Plattner et al., 2006). It has been reported that increased phosphorylation of the Ser202/Thr205 (AT8) site decreased mitochondrial transport in axons in $\mathrm{AD}$, leading to axonal degeneration (Shahpasand et al., 2012). Recent study has shown that tau phosphorylation at Ser 396 is markedly increased in hippocampus of $5 \times$ FAD mice compared with wild type mice. It occurs earlier than the appearance of learning and memory disorders (Kanno et al., 2014). Moreover, phosphorylated tau subsequently aggregates to form paired helical filaments (PHFs) and further forms NFTs, which lead to neuronal dysfunction and loss. The density of NFTs is directly correlated with disease severity and the degree of dementia in $\mathrm{AD}$ patients (Alonso et al., 1996; Ballatore et al., 2007). Therefore, phosphorylated tau has become a novel therapeutic target in $\mathrm{AD}$. In the current study, we observed that MenSC transplantation dramatically reduced tau phosphorylation at Ser202/Thr205 (AT8) and Ser396 sites in the brains of APP/PS1 mice (Figures 7A-D). These data strongly demonstrate that MenSC transplantation can inhibit tau phosphorylation in APP/PS1 mice.

Several kinases have been reported to phosphorylate tau. Glycogen synthase kinase-3 $\beta$ (GSK3 $\beta$ ) is one of the most important kinases due to its ability to phosphorylate tau at the majority of its serine/threonine sites, which cause associated toxicities in AD (Medina et al., 2011; Llorens-Martín et al., 2014). GSK3 $\beta$ leads to tau hyperphosphorylation at many sites in vitro (Rankin et al., 2007) and in vivo (Liu et al., 2003). Recent studies have been reported that Ser396 is a major substrate for GSK$3 \beta$ (Liu et al., 2007) and Ser202/Thr205 (AT8) is one of the moderate phosphorylation sites for GSK-3 $\beta$ (Illenberger et al., 1998). Researchers also have found that several small molecule inhibitors of GSK-3 $\beta$ could reduce tau phosphorylation at Ser396 in vivo (Selenica et al., 2007). Overexpression of GSK-3 $\beta$ in transgenic mice leads to tau phosphorylation (Engel et al., 2006), and GSK-3 $\beta$ inhibitors significantly alleviate tau phosphorylation and tau-induced neurodegeneration (Noble et al., 2005). The activity of GSK-3 $\beta$ is regulated by autophosphorylation at Ser9, which results in a kinase-inactive state (Medina et al., 2011). In the previous study, our group demonstrated that MenSCs could inactivate GSK-3 $\beta$ in order to promote the repair of damaged lung tissue in acute lung injury (Xiang et al., 2017). Therefore, GSK-3 $\beta$ was selected in our study. We found that MenSC transplantation markedly upregulated the phosphorylation of GSK-3 $\beta$ at Ser9, which resulted in GSK-3 $\beta$ inactivity in both the hippocampus and the cortex of APP/PS1 mice (Figures 7E,F). These results indicate that MenSC transplantation could alleviate tau phosphorylation in the brains of APP/PS1 mice, which is associated with the suppression of GSK-3 $\beta$ activity. Moreover, GSK-3 $\beta$ is reported not only to phosphorylate tau, but also to regulate the production of $A \beta$. GSK- $3 \beta$ could regulate $\beta$-secretase (BACE1) expression to control $A \beta$ formation. In the amyloidogenic pathway, APP is cleaved by $\beta$-secretase (BACE1) to generate $\beta$-CTF. Subsequently, $\gamma$-secretase is cleaved $\beta$-CTF to release $A \beta$, which tends to aggregate to form senile plaques. It has been reported that GSK-3 $\beta$ inhibition reduced BACE1-mediated cleavage of APP through NF- $\kappa$ B signal pathway in the APP/PS1 transgenic mouse model (Ly et al., 2013), further supporting that the inhibition of GSK-3 $\beta$ reduces $A \beta$ pathology. Our results have shown that MenSCs transplantation reduced BACE1 expression and $\beta$-CTF in APP/PS1 mice (Figure 4), which is possibly related to inactivate GSK-3 $\beta$. Accumulating evidence suggest that GSK-3 $\beta$ affects inflammatory. Several pro-inflammatory cytokines such as TNF have been found to activate GSK-3 $\beta$, whereas GSK-3 $\beta$ inhibitors could protect from inflammatory conditions in animal models (Wyss-Coray and Rogers, 2012). IL-1 $\beta$ has been shown to increase tau phosphorylation through increasing GSK-3 $\beta$ and MAPK (Ghosh et al., 2013). These evidence demonstrate that GSK-3 $\beta$ plays a pivotal and central role in the pathogenesis of AD.

Excessive $A \beta$ accumulation and hyperphosphorylated tau are associated with cognitive decline and memory deficits in AD model mice (Chen et al., 2000). The extent to which MenSC transplantation can attenuate cognitive impairment was studied here using the Morris water maze. In our study, MenSC transplantation resulted in improved spatial learning of APP/PS1 mice. Furthermore, the WT and MenSC-treated APP/PS1 mice showed a target-oriented swimming pattern, while the PBStreated APP/PS1 mice swam in circles. These results suggest that the PBS-treated APP/PS1 mice reached the platform randomly and demonstrate that MenSC treatment dramatically attenuated cognitive decline of APP/PS1 mice.

\section{CONCLUSION}

In conclusion, our results show that MenSC transplantation can ameliorate $\mathrm{AD}$ pathology and cognitive deficits in a transgenic mouse model of AD. However, the mechanisms of the MenSCmediated improvements in APP/PS1 mice remain obscure. Most recent studies have considered that the neuroprotective effects of MSC transplantation are mainly stimulated by the paracrine 
effect (Drago et al., 2013). Our group also demonstrated that the beneficial effects of MenSC transplantation in mice with liver fibrosis and type 1 diabetes are mainly promoted by paracrine effects (Wu et al., 2014; Chen et al., 2017b). More research will be aimed at elaborating the exact paracrine effects of MenSCs and determining which factors secreted by MenSCs are capable of alleviating $\mathrm{AD}$ pathology and inducing activated microglia phenotype turn-over toward neuroprotection.

Here, we show that intracerebral MenSC transplantation reduces $\mathrm{A} \beta$ deposition mediated by BACE1 activity and $A \beta$-degrading enzymes, attenuates tau phosphorylation mediated by GSK3 $\beta$, induces activated microglia phenotype turn-over toward neuroprotection and improves cognitive decline in APP/PS1 mice. These data indicate that MenSCs may be a novel cell therapy for $\mathrm{AD}$.

\section{REFERENCES}

Alcayaga-Miranda, F., Cuenca, J., Luz-Crawford, P., Aguila-Díaz, C., Fernandez, A., Figueroa, F. E., et al. (2015). Characterization of menstrual stem cells: angiogenic effect, migration and hematopoietic stem cell support in comparison with bone marrow mesenchymal stem cells. Stem Cell Res. Ther. 6:32. doi: 10.1186/s13287-015-0013-5

Allickson, J., and Xiang, C. (2012). Human adult stem cells from menstrual blood and endometrial tissue. J. Zhejiang Univ. Sci. B 13, 419-420. doi: 10.1631/jzus. B1200062

Alonso, A. C., Grundke-Iqbal, I., and Iqbal, K. (1996). Alzheimer's disease hyperphosphorylated tau sequesters normal tau into tangles of filaments and disassembles microtubules. Nat. Med. 2, 783-787. doi: 10.1038/nm0796-783

Alzheimer's Association (2011). 2011 Alzheimer's disease facts and figures. Alzheimers Dement. 7, 208-244. doi: 10.1016/j.jalz.2011.02.004

Ballard, C., Gauthier, S., Corbett, A., Brayne, C., Aarsland, D., and Jones, E. (2011). Alzheimer's disease. Lancet 377, 1019-1031. doi: 10.1016/S0140-6736(10) 61349-9

Ballatore, C., Lee, V. M., and Trojanowski, J. Q. (2007). Tau-mediated neurodegeneration in Alzheimer's disease and related disorders. Nat. Rev. Neurosci. 8, 663-672. doi: 10.1038/nrn2194

Boche, D., Perry, V. H., and Nicoll, J. A. (2013). Review: activation patterns of microglia and their identification in the human brain. Neuropathol. Appl. Neurobiol. 39, 3-18. doi: 10.1111/nan.12011

Chen, G., Chen, K. S., Knox, J., Inglis, J., Bernard, A., Martin, S. J., et al. (2000). A learning deficit related to age and beta-amyloid plaques in a mouse model of Alzheimer's disease. Nature 408, 975-979. doi: 10.1038/35050103

Chen, L., Xiang, B., Wang, X., and Xiang, C. (2017a). Exosomes derived from human menstrual blood-derived stem cells alleviate fulminant hepatic failure. Stem Cell Res. Ther. 8:9. doi: 10.1186/s13287-016-0453-6

Chen, L., Zhang, C., Chen, L., Wang, X., Xiang, B., Wu, X., et al. (2017b). Human menstrual blood-derived stem cells ameliorate liver fibrosis in mice by targeting hepatic stellate cells via paracrine mediators. Stem Cells Transl. Med. 6, 272-284. doi: 10.5966/sctm.2015-0265

Cunningham, C. (2013). Microglia and neurodegeneration: the role of systemic inflammation. Glia 61, 71-90. doi: 10.1002/glia.22350

Doody, R. S., Thomas, R. G., Farlow, M., Iwatsubo, T., Vellas, B., Joffe, S., et al. (2014). Phase 3 trials of solanezumab for mild-to-moderate Alzheimer's disease. N. Engl. J. Med. 370, 311-321. doi: 10.1056/NEJMoa1312889

Drago, D., Cossetti, C., Iraci, N., Gaude, E., Musco, G., Bachi, A., et al. (2013). The stem cell secretome and its role in brain repair. Biochimie 95, 2271-2285. doi: 10.1016/j.biochi.2013.06.020

Engel, T., Hernandez, F., Avila, J., and Lucas, J. J. (2006). Full reversal of Alzheimer's disease-like phenotype in a mouse model with conditional overexpression of glycogen synthase kinase 3. Neuroscientist 26, 5083-5090. doi: 10.1523/ JNEUROSCI.0604-06.2006

Fang, Y., Yao, L., Li, C., Wang, J., Wang, J., Chen, S., et al. (2016). The blockage of the $\mathrm{Nogo} / \mathrm{NgR}$ signal pathway in microglia alleviates the formation

\section{AUTHOR CONTRIBUTIONS}

$\mathrm{YZ}$ and $\mathrm{CX}$ designed the research work. YZ performed the intracerebral transplantation of MenSCs. YZ, XC, and YiW performed the Morris water maze analyses and biochemical analyses. YZ, YaW, and YL performed the RT-PCR analyses and histopathological analyses. YZ and XC interpreted the data and drafted the manuscript. All authors read and approved the final manuscript.

\section{FUNDING}

This study was supported by grants from the National Key R\&D Program of China (Grant No. 2017YFA0105701).

of $\mathrm{A} \beta$ plaques and tau phosphorylation in APP/PS1 transgenic mice. J. Neuroinflammation 13:56. doi: 10.1186/s12974-016-0522-x

Farris, W., Mansourian, S., Chang, Y., Lindsley, L., Eckman, E. A., Frosch, M. P., et al. (2003). Insulin-degrading enzyme regulates the levels of insulin, amyloid beta-protein, and the beta-amyloid precursor protein intracellular domain in vivo. Proc. Natl. Acad. Sci. U.S.A. 100, 4162-4167. doi: 10.1073/pnas. 0230450100

Fukumoto, H., Rosene, D. L., Moss, M. B., Raju, S., Hyman, B. T., and Irizarry, M. C. (2004). $\beta$-secretase activity increases with aging in human, monkey, and mouse brain. Am. J. Pathol. 164, 719-725. doi: 10.1016/S0002-9440(10)63159-8

Gauthier, S., Feldman, H. H., Schneider, L. S., Wilcock, G. K., Frisoni, G. B., Hardlund, J. H., et al. (2016). Efficacy and safety of tau-aggregation inhibitor therapy in patients with mild or moderate Alzheimer's disease: a randomised, controlled, double-blind, parallel-arm, phase 3 trial. Lancet 388, 2873-2884. doi: 10.1016/S0140-6736(16)31275-2

Ghosh, S., Wu, M. D., Shaftel, S. S., Kyrkanides, S., LaFerla, F. M., Olschowka, J. A., et al. (2013). Sustained interleukin-1 $\beta$ overexpression exacerbates tau pathology despite reduced amyloid burden in an Alzheimer's mouse model. J. Neurosci. 33, 5053-5064. doi: 10.1523/JNEUROSCI.4361-12.2013

Götz, J., Chen, F., van Dorpe, J., and Nitsch, R. M. (2001). Formation of neurofibrillary tangles in P301l tau transgenic mice induced by Abeta 42 fibrils. Science 293, 1491-1495. doi: 10.1126/science.1062097

Heneka, M. T., Kummer, M. P., and Latz, E. (2014). Innate immune activation in neurodegenerative disease. Nat. Rev. Immunol. 14, 463-477. doi: 10.1038/ nri3705

Hickman, S. E., Allison, E. K., and El Khoury, J. (2008). Microglial dysfunction and defective beta-amyloid clearance pathways in aging Alzheimer's disease mice. J. Neurosci. 28, 8354-8360. doi: 10.1523/JNEUROSCI.0616-08.2008

Illenberger, S., Zheng-Fischhöfer, Q., Preuss, U., Stamer, K., Baumann, K., Trinczek, B., et al. (1998). The endogenous and cell cycle-dependent phosphorylation of tau protein in living cells: implications for Alzheimer's disease. Mol. Biol. Cell 9, 1495-1512. doi: 10.1091/mbc.9.6.1495

Iwata, N., Tsubuki, S., Takaki, Y., Shirotani, K., Lu, B., Gerard, N. P., et al. (2001). Metabolic regulation of brain Abeta by neprilysin. Science 292, 1550-1552. doi: 10.1126/science.1059946

Kanno, T., Tsuchiya, A., and Nishizaki, T. (2014). Hyperphosphorylation of Tau at Ser396 occurs in the much earlier stage than appearance of learning and memory disorders in 5XFAD mice. Behav. Brain Res. 274, 302-306. doi: 10.1016/j.bbr.2014.08.034

Khoury, M., Alcayaga-Miranda, F., Illanes, S. E., and Figueroa, F. E. (2014). The promising potential of menstrual stem cells for antenatal diagnosis and cell therapy. Front. Immunol. 5:205. doi: 10.3389/fimmu.2014.00205

Kim, J. Y., Kim, D. H., Kim, J. H., Lee, D., Jeon, H. B., Kwon, S. J., et al. (2012). Soluble intracellular adhesion molecule- 1 secreted by human umbilical cord blood-derived mesenchymal stem cell reduces amyloid- $\beta$ plaques. Cell Death Differ. 19, 680-691. doi: 10.1038/cdd.2011.140

Lai, D., Wang, F., Yao, X., Zhang, Q., Wu, X., and Xiang, C. (2015). Human endometrial mesenchymal stem cells restore ovarian function through 
improving the renewal of germline stem cells in a mouse model of premature ovarian failure. J. Transl. Med. 13:155. doi: 10.1186/s12967-015-0516-y

Latta, C. H., Sudduth, T. L., Weekman, E. M., Brothers, H. M., Abner, E. L., Popa, G. J., et al. (2015). Determining the role of IL-4 induced neuroinflammation in microglial activity and amyloid- $\beta$ using BV2 microglial cells and APP/PS1 transgenic mice. J. Neuroinflamm. 12:41. doi: 10.1186/s12974-015-0243-6

Lee, H. J., Lee, J. K., Lee, H., Carter, J. E., Chang, J. W., Oh, W., et al. (2012). Human umbilical cord blood-derived mesenchymal stem cells improve neuropathology and cognitive impairment in an Alzheimer's disease mouse model through modulation of neuroinflammation. Neurobiol. Aging 33, 588-602. doi: 10.1016/ j.neurobiolaging.2010.03.024

Lee, J. K., Jin, H. K., Endo, S., Schuchman, E. H., Carter, J. E., and Bae, J. S. (2010). Intracerebral transplantation of bone marrow-derived mesenchymal stem cells reduces amyloid-beta deposition and rescues memory deficits in Alzheimer's disease mice by modulation of immune responses. Stem Cells 28, 329-343. doi: $10.1002 /$ stem.277

Lee, J. K., Schuchman, E. H., Jin, H. K., and Bae, J. S. (2012). Soluble CCL5 derived from bone marrow-derived mesenchymal stem cells and activated by amyloid $\beta$ ameliorates Alzheimer's disease in mice by recruiting bone marrow-induced microglia immune responses. Stem Cells 30, 1544-1555. doi: 10.1002/stem.1125

Liu, F., Li, B., Tung, E. J., Grundke-Iqbal, I., Iqbal, K., and Gong, C. X. (2007). Site-specific effects of tau phosphorylation on its microtubule assembly activity and self-aggregation. Eur. J. Neurosci. 26, 3429-3436. doi: 10.1111/j.1460-9568. 2007.05955.x

Liu, S. J., Zhang, A. H., Li, H. L., Wang, Q., Deng, H. M., Netzer, W. J., et al. (2003). Overactivation of glycogen synthase kinase- 3 by inhibition of phosphoinositol3 kinase and protein kinase $\mathrm{C}$ leads to hyperphosphorylation of tau and impairment of spatial memory. J. Neurochem. 87, 1333-1344. doi: 10.1046/j. 1471-4159.2003.02070.x

Liu, Y., Liu, X., Hao, W., Decker, Y., Schomburg, R., Fülöp, L., et al. (2014). IKK $\beta$ deficiency in myeloid cells ameliorates Alzheimer's disease-related symptoms and pathology. J. Neurosci. 34, 12982-12999. doi: 10.1523/JNEUROSCI.134814.2014

Llorens-Martín, M., Jurado, J., Hernández, F., and Avila, J. (2014). GSK-3 $\beta$, a pivotal kinase in Alzheimer disease. Front. Mol. Neurosci. 7:46. doi: 10.3389/ fnmol.2014.00046

Lovestone, S., Boada, M., Dubois, B., Hüll, M., Rinne, J. O., Huppertz, H. J., et al. (2015). A phase II trial of tideglusib in Alzheimer's disease. J. Alzheimers Dis. 45, 75-88. doi: 10.3233/JAD- 141959

Ly, P. T., Wu, Y., Zou, H., Wang, R., Zhou, W., Kinoshita, A., et al. (2013). Inhibition of GSK3beta-mediated BACE1 expression reduces Alzheimerassociated phenotypes. J. Clin. Invest. 123, 224-235. doi: 10.1172/JCI64516

Ma, T., Gong, K., Ao, Q., Yan, Y., Song, B., Huang, H., et al. (2013). Intracerebral transplantation of adipose-derived mesenchymal stem cells alternatively activates microglia and ameliorates neuropathological deficits in Alzheimer's disease mice. Cell Transplant. 22, S113-S126. doi: 10.3727/096368913X672181

Medina, M., Garrido, J. J., and Wandosell, F. G. (2011). Modulation of GSK3 as a therapeutic strategy on Tau pathologies. Front. Mol. Neurosci. 4:24. doi: $10.3389 / \mathrm{fnmol} .2011 .00024$

Meng, X., Ichim, T. E., Zhong, J., Rogers, A., Yin, Z., Jackson, J., et al. (2007). Endometrial regenerative cells: a novel stem cell population. J. Transl. Med. 5:57. doi: 10.1186/1479-5876-5-57

Noble, W., Planel, E., Zehr, C., Olm, V., Meyerson, J., Suleman, F., et al. (2005). Inhibition of glycogen synthase kinase- 3 by lithium correlates with reduced tauopathy and degeneration in vivo. Proc. Natl. Acad. Sci. U.S.A. 102, 69906995.

Ohno, M., Sametsky, E. A., Younkin, L. H., Oakley, H., Younkin, S. G., Citron, M., et al. (2004). BACE1 deficiency rescues memory deficits and cholinergic dysfunction in a mouse model of Alzheimer's disease. Neuron 41, 27-33. doi: 10.1016/S0896-6273(03)00810-9

Pimplikar, S. W. (2014). Neuroinflammation in Alzheimer's disease: from pathogenesis to a therapeutic target. J. Clin. Immunol. 34, S64-S69. doi: $10.1007 / \mathrm{s} 10875-014-0032-5$

Plattner, F., Angelo, M., and Giese, K. P. (2006). The roles of cyclin-dependent kinase 5 and glycogen synthase kinase 3 in tau hyperphosphorylation. J. Biol. Chem. 281, 25457-25465. doi: 10.1074/jbc.M603469200
Rankin, C. A., Sun, Q., and Gamblin, T. C. (2007). Tau phosphorylation by GSK3beta promotes tangle-like filament morphology. Mol. Neurodegener. 2:12. doi: $10.1186 / 1750-1326-2-12$

Ransohoff, R. M. (2016). How neuroinflammation contributes to neurodegeneration. Science 353, 777-783. doi: 10.1126/science.aag2590

Salloway, S., Sperling, R., Fox, N. C., Blennow, K., Klunk, W., Raskind, M., et al. (2014). Two phase 3 trials of bapineuzumab in mild-to-moderate Alzheimer's disease. N. Engl. J. Med. 370, 322-333. doi: 10.1056/NEJMoa1304839

Selenica, M. L., Jensen, H. S., Larsen, A. K., Pedersen, M. L., Helboe, L., Leist, M., et al. (2007). Efficacy of small-molecule glycogen synthase kinase-3 inhibitors in the postnatal rat model of tau hyperphosphorylation. Br. J. Pharmacol. 152, 959-979. doi: 10.1038/sj.bjp.0707471

Shahpasand, K., Uemura, I., Saito, T., Asano, T., Hata, K., Shibata, K., et al. (2012). Regulation of mitochondrial transport and inter-microtubule spacing by tau phosphorylation at the sites hyperphosphorylated in Alzheimer's disease. J. Neurosci. 32, 2430-2441. doi: 10.1523/JNEUROSCI.5927-11.2012

Spillantini, M. G., and Goedert, M. (2013). Tau pathology and neurodegeneration. Lancet Neurol. 12, 609-622. doi: 10.1016/S1474-4422(13)70090-5

Sugawara, K., Hamatani, T., Yamada, M., Ogawa, S., Kamijo, S., Kuji, N., et al. (2014). Derivation of human decidua-like cells from amnion and menstrual blood. Sci. Rep. 4:4599. doi: 10.1038/srep04599

Tuppo, E. E., and Arias, H. R. (2005). The role of inflammation in Alzheimer's disease. Int. J. Biochem. Cell Biol. 37, 289-305. doi: 10.1016/j.biocel.2004.07.009

Wolff, E. F., Gao, X. B., Yao, K. V., Andrews, Z. B., Du, H., Elsworth, J. D., et al. (2011). Endometrial stem cell transplantation restores dopamine production in a Parkinson's disease model. J. Cell. Mol. Med. 15, 747-755. doi: 10.1111/j.15824934.2010.01068.x

Wu, X., Luo, Y., Chen, J., Pan, R., Xiang, B., Du, X., et al. (2014). Transplantation of human menstrual blood progenitor cells improves hyperglycemia by promoting endogenous progenitor differentiation in type 1 diabetic mice. Stem Cells Dev. 23, 1245-1257. doi: 10.1089/scd.2013.0390

Wyss-Coray, T., and Rogers, J. (2012). Inflammation in Alzheimer disease-a brief review of the basic science and clinical literature. Cold Spring Harb. Perspect. Med. 2:a006346. doi: 10.1101/cshperspect.a006346

Xiang, B., Chen, L., Wang, X., Zhao, Y., Wang, Y., and Xiang, C. (2017). Transplantation of menstrual blood-derived mesenchymal stem cells promotes the repair of LPS-induced acute lung injury. Int. J. Mol. Sci. 18:689. doi: 10.3390/ ijms18040689

Yang, L. B., Lindholm, K., Yan, R., Citron, M., Xia, W., Yang, X. L., et al. (2003). Elevated beta-secretase expression and enzymatic activity detected in sporadic Alzheimer disease. Nat. Med. 9, 3-4. doi: 10.1038/nm0103-3

Ye, X., and Cai, Q. (2014). Snapin-mediated BACE1 retrograde transport is essential for its degradation in lysosomes and regulation of APP processing in neurons. Cell Rep. 6, 24-31. doi: 10.1016/j.celrep.2013.12.008

Zempel, H., Luedtke, J., Kumar, Y., Biernat, J., Dawson, H., Mandelkow, E., et al. (2013). Amyloid- $\beta$ oligomers induce synaptic damage via tau-dependent microtubule severing by TTLL6 and spastin. EMBO J. 32, 2920-2937. doi: 10.1038/emboj.2013.207

Zhang, Y., Thompson, R., Zhang, H., and Xu, H. (2011). APP processing in Alzheimer's disease. Mol. Brain 4:3. doi: 10.1186/1756-6606-4-3

Zhang, Z., Wang, J. A., Xu, Y., Jiang, Z., Wu, R., Wang, L., et al. (2013). Menstrual blood derived mesenchymal cells ameliorate cardiac fibrosis via inhibition of endothelial to mesenchymal transition in myocardial infarction. Int. J. Cardiol. 168, 1711-1714. doi: 10.1016/j.ijcard.2013.03.126

Conflict of Interest Statement: The authors declare that the research was conducted in the absence of any commercial or financial relationships that could be construed as a potential conflict of interest.

Copyright ( $\odot 2018$ Zhao, Chen, Wu, Wang, Li and Xiang. This is an open-access article distributed under the terms of the Creative Commons Attribution License (CC BY). The use, distribution or reproduction in other forums is permitted, provided the original author(s) and the copyright owner are credited and that the original publication in this journal is cited, in accordance with accepted academic practice. No use, distribution or reproduction is permitted which does not comply with these terms. 